\title{
Longitudinal changes in health-related quality of life according to clinical course among patients with non-tuberculous mycobacterial pulmonary disease: a prospective cohort study
}

\author{
Nakwon Kwak', Sung A Kim², Sun Mi Choi', Jinwoo Lee ${ }^{1}$, Chang-Hoon Lee ${ }^{1}$ and Jae-Joon Yim ${ }^{\text {1* }}$
}

\begin{abstract}
Background: Improvement in health-related quality of life (HRQL) has been suggested as an alternative treatment goal of non-tuberculous mycobacterial pulmonary disease (NTM-PD). This study was performed to elucidate the longitudinal changes in HRQL using St. George's Respiratory Questionnaire (SGRQ) among patients with NTM-PD according to their clinical course.

Methods: Patients with NTM-PD who participated in Seoul National University Hospital's prospective NTM cohort were screened. Participants for whom the SGRQ score was estimated with the one-year interval for $\geq$ three times were included. The longitudinal trends of the SGRQ score were assessed. The impact of the clinical course on the change in the SGRQ score was elucidated using multilevel mixed-effects linear regression with a repeated-measures model.

Results: In total, 114 patients were analyzed. During the median 5-year observation period, 53 patients started antimycobacterial treatment and 61 patients were observed without treatment. Among the treated patients, 24 (45.2\%) achieved microbiological cure. Patients who required treatment eventually had worsening SGRQ scores with time compared with patients who could be observed without treatment $(P<0.001)$. In cured patients, the SGRQ score decreased from 33.9 at baseline to 20.8 at 1 year post-treatment $(P<0.001), 21.3$ at 2 years $(P<0.001)$, and 17.6 at 3 years $(P<0.001)$. The SGRQ scores also decreased for 2 years of treatment in patients with NTM-PD that could not be cured, although this decrease did not last for 3 years of treatment.
\end{abstract}

Conclusion: Worsening HRQL scores were associated with the initiation of treatment and, in turn, treatment improved HRQL scores of patients with NTM-PD.

Trial registration: This study was registered to the ClinicalTrials.gov (Identifier: NCT01616745 / registration date: June 12, 2012). The protocol was retrospectively registered.

Keywords: Health-related quality of life, St. George's respiratory questionnaire, Non-tuberculous mycobacteria

\footnotetext{
*Correspondence: yimjj@snu.ac.kr

'Division of Pulmonary and Critical Care Medicine, Department of Internal

Medicine, Seoul National University College of Medicine, 101 Daehak-Ro,

Jongno-Gu, Seoul 110-744, South Korea

Full list of author information is available at the end of the article
}

(c) The Author(s). 2020 Open Access This article is licensed under a Creative Commons Attribution 4.0 International License, which permits use, sharing, adaptation, distribution and reproduction in any medium or format, as long as you give appropriate credit to the original author(s) and the source, provide a link to the Creative Commons licence, and indicate if changes were made. The images or other third party material in this article are included in the article's Creative Commons licence, unless indicated otherwise in a credit line to the material. If material is not included in the article's Creative Commons licence and your intended use is not permitted by statutory regulation or exceeds the permitted use, you will need to obtain permission directly from the copyright holder. To view a copy of this licence, visit http://creativecommons.org/licenses/by/4.0/ The Creative Commons Public Domain Dedication waiver (http://creativecommons.org/publicdomain/zero/1.0/) applies to the data made available in this article, unless otherwise stated in a credit line to the data. 


\section{Background}

The burden of non-tuberculous mycobacterial pulmonary disease (NTM-PD) has been increasing globally. An increased incidence of NTM-PD has been reported in East Asia [1, 2], the United States [3], and Europe [4]. This phenomenon has also been observed in South Korea. According to a population-based study, the incidence of NTM-PD in South Korea increased from 6.0 cases/100,000 population per year to 19.0 cases/100,000 population per year from 2007 to 2016 [5].

Although the burden of NTM-PD has steadily increased, treatment of NTM-PD is complicated and the outcome is unsatisfactory. Treatment of NTM-PD requires the use of several antibiotics for up to 2 years [ 6 , 7]. However, the treatment success rate was only $60.0 \%$ for Mycobacterium avium complex (MAC) pulmonary disease [8] and $33.0-41.2 \%$ for $M$. abscessus complex pulmonary disease [9-11]. Moreover, microbiologic recurrence is observed among half of patients who have completed treatment for MAC pulmonary disease [12].

Because of the difficulties in eradicating NTM-PD, alternative treatment goals have been suggested. These goals encompass improvements in symptoms, radiographic lesions, and quality of life $[6,13]$. In fact, the St. George's Respiratory Questionnaire (SGRQ) score, which is the most commonly adopted measure of health-related quality of life (HRQL), has been validated for patients with NTM-PD. [14, 15]. The SGRQ score, the decline of which means the improvement of HRQL, was reported to be higher among patients with NTMPD [16] and has been suggested as an indicator of the treatment response in patients with $M$. abscessus complex pulmonary disease [13]. However, the longitudinal changes in HRQL among patients with NTM-PD have not been fully evaluated. Through this study, we aimed to elucidate the longitudinal changes in HRQL using the SGRQ among patients with NTM-PD according to their clinical course.

\section{Methods}

\section{Study participants}

Patients with NTM-PD who participated in the ongoing prospective Seoul National University Hospital NTM cohort (ClinicalTrials.gov identifier: NCT01616745), which commenced on 1 July 2011, were included in this analysis $[17,18]$. The diagnosis of NTM-PD followed the criteria suggested by the American Thoracic Society/Infectious Diseases Society of America [6] and British Thoracic Society guidelines [7]. Patients who completed the SGRQ at least three times with the one-year interval were included in this analysis. Patients with loss to follow-up were excluded. All participants in this cohort were newly diagnosed as having NTM-PD and were treatment-naïve. All patients included in the analysis provided written consents for the study. This study was conducted in accordance with the amended Declaration of Helsinki and the Institutional Review Board of Seoul National University Hospital approved the protocol (IRB No. 1809-112-974).

\section{Baseline and follow-up evaluations}

All patients' demographic, clinical, and laboratory data were collected and analyzed at the time of study enrollment. Chest computed tomography was performed, and radiographic findings were analyzed. After enrollment, the patients were followed up every 3 to 6 months. On each visit, the participants submitted sputum specimens for acid-fast bacilli smears and mycobacterial cultures and underwent simple chest radiographs. When NTM was isolated from respiratory specimens, the mycobacterial species were identified using the $16 \mathrm{~S}$ rRNA [19] and $r p o B$ gene sequencing [20, 21].

The decision to initiate treatment in each patient was made by the on-duty physicians based on clinical deterioration (e.g., new-onset hemoptysis) or radiographic deterioration defined by two independent chest radiologists (e.g., cavity formation).

\section{Treatment}

The treatment regimens were in accordance with the American Thoracic Society/Infectious Diseases Society of America guideline [6] and were adjusted based on the results of drug susceptibility tests, patients' tolerance, and adverse events. Generally, patients with MAC infection were treated with a macrolide, rifampicin, and ethambutol. The use of amikacin was determined by the on-duty physicians. Once a decision had been made regarding the use of amikacin, the patients were initially hospitalized for at least 2 to 3 weeks, and the maintenance of injectable drugs was prolonged according to the clinical response and adverse events. For patients with $M$. abscessus complex infection, the combination of antibiotic therapy including a macrolide and at least two parenteral agents including amikacin, cefoxitin, or imipenem were administered as previously described [22]. Once treatment was initiated, the patients were followed up every 4 to 8 weeks.

\section{SGRQ scoring}

The Korean-translated SGRQ, which measures HRQL in patients with diseases of airway obstruction using 76 weighted responses, has been proven reliable and valid for various chronic respiratory diseases [23]. The scores were calculated individually for symptoms, activity, and impacts; the total score was also calculated. The SGRQ was repeatedly performed each year from the time of cohort enrollment among the participants in the present study. The questionnaire performed with an interval of 
10 to 14 months were allowed for the analysis. When the patients received antibiotic treatment for NTM-PD, the questionnaire submitted up to 2 months before the time of treatment initiation was adopted for the study.

\section{Statistical analysis}

The patients were categorized into two groups: those who could be observed without treatment and those who needed the treatment for NTM-PD during the follow-up period. The latter group was re-classified into two groups according to the treatment outcomes: 1) microbiological cure, which was defined as three or more consecutive negative and no positive cultures of the causative species from respiratory samples after culture conversion and until the end of anti-mycobacterial treatment [24], and 2) treatment failure, which was defined as the re-emergence of two or more positive cultures or persistence of positive cultures of the causative species from respiratory samples after $\geq 12$ months of anti-mycobacterial treatment, while the patient was still undergoing treatment [24].

The impact of covariates including SGRQ score on treatment initiation was analyzed. In addition, the longitudinal changes in the SGRQ score were compared between the patients who could be observed without treatment and the patients who required treatment later. Once treatment was initiated, the longitudinal trend was also compared between the patients who achieved microbiological cure and the patients who had treatment failure.

Data are expressed as median with interquartile range (IQR) for continuous variables and as proportion for categorical variables. Wilcoxon's rank-sum test and Fisher's exact test were used to compare continuous and categorical variables among different groups, respectively. To determine the covariates associated with treatment initiation, multivariate logistic regression egression analysis was adopted. Multilevel mixed-effects regression was used to estimate the effect of each variable on the longitudinal change in the SGRQ score and the predictive means of the SGRQ score at each year. All analyses were performed using STATA version 14.2 (StataCorp, College Station, TX, USA).

\section{Results}

\section{Patient characteristics}

During the study period, a total of 373 patients was enrolled in the cohort. Sixty one patients who were lost to follow-up, 126 patients who submitted SGRQ questionnaire irregularly, and 72 patients who submitted questionnaire twice or less were excluded for the analysis. Finally, 114 patients and 504 questionnaires were included in the analysis (Fig. 1). Their median age was 63 years (IQR, 57-70 years), and 83 patients were female

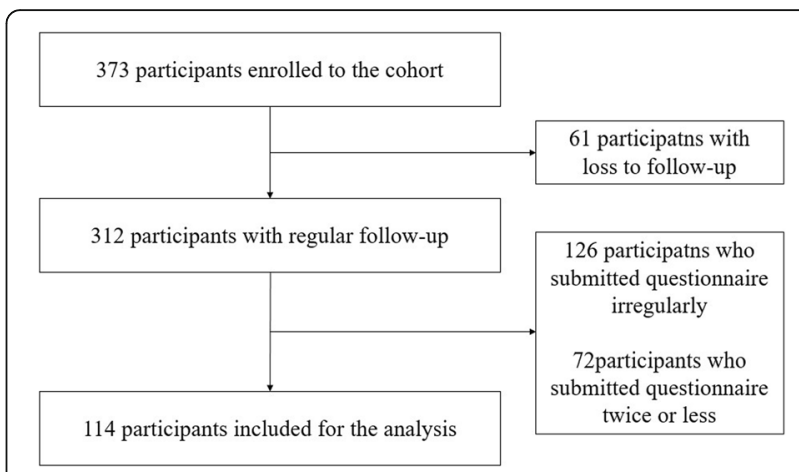

Fig. 1 Flow diagram for patients' inclusion

(72.8\%). MAC (74.6\%) was the most common pathogen, followed by $M$. abscessus complex (24.6\%). The median follow-up duration was 5.0 years (IQR, 4.0-6.0 years). Sixty one patients were regularly monitored without treatment, while 53 patients received anti-mycobacterial treatment. The median interval from enrollment to treatment initiation was 12.0 months (IQR, 2.0-24.0 months). Among the treated patients, 24 (45.3\%) achieved microbiologic cure.

The median age, sex proportion, smoking habits, comorbidities, and distribution of etiologic nontuberculous mycobacteria were not different between patients observed without treatment and those who needed treatment eventually. However, the body mass index (BMI) was higher in patients observed without treatment $(P=0.040)$, while fibrocavitary form was more common in patients who received treatment $(P=0.017)$. Among treated patients, the patients who achieved cure had a higher BMI than those who experienced treatment failure $(P=0.024)$ (Table 1$)$.

\section{Changes in SGRQ score during observation}

In total, 504 questionnaires were obtained from 114 patients (median, 4 [IQR, 4-5] per patient). When the longitudinal changes of SGRQ scores were compared, patients who eventually required treatment had worsening SGRQ values compared with patients who could be observed without treatment (coefficient, 4.61; $P<0.001$ ). While the initial SGRQ scores did not differ between patients who could be observed and patients who eventually required treatment (mean difference, 1.62; 95\% confidence interval $[\mathrm{CI}],-5.93$ to 9.17 ), the difference between them widened over time (Fig. 2). According to multivariable logistic analysis, changes in SGRQ scores, but not baseline SGRQ, were associated with initiation of treatment (Table 2).

During the observation period, 17 patients among untreated group achieved spontaneous culture conversion, defined as three or more consecutive negative cultures. 
Table 1 Baseline characteristics of 114 patients with NTM pulmonary disease according to their clinical course

\begin{tabular}{|c|c|c|c|}
\hline & Patients who & Patients who needed treatm & $(n=53)$ \\
\hline & $\begin{array}{l}\text { were observed } \\
\text { without } \\
\text { treatment ( } n= \\
61 \text { ) }\end{array}$ & Microbiologic cure $(n=24)$ & Treatment failure $(n=29)$ \\
\hline Age, years & $64(54-72)$ & $61(54-70)$ & $63(57-70)$ \\
\hline Female sex & $40(65.6)$ & $19(79.2)$ & $24(82.8)$ \\
\hline $\mathrm{BMI}, \mathrm{kg} / \mathrm{m}^{2 *}$ & $21.4(19.8-22.9)$ & $21.1(19.9-22.1)$ & $19.4(17.8-21.4)$ \\
\hline Current/former smoker, n (\%) & $14(23.0)$ & $6(25.0)$ & $4(13.8)$ \\
\hline Comorbidities, n (\%) & & & \\
\hline History of tuberculosis & $23(37.7)$ & $10(41.7)$ & $9(31.0)$ \\
\hline Diabetes mellitus & $4(6.6)$ & $2(8.3)$ & $2(6.9)$ \\
\hline Malignancy & $6(9.8)$ & $3(12.5)$ & $3(10.3)$ \\
\hline COPD & $8(13.1)$ & $3(12.5)$ & $2(6.9)$ \\
\hline Radiographic features, n (\%) & & & \\
\hline Non-cavitary nodular bronchiectatic & $51(83.7)$ & $16(66.7)$ & $18(62.1)$ \\
\hline Cavitary nodular bronchiectatic & $7(11.5)$ & $3(12.5)$ & $6(20.7)$ \\
\hline Fibrocavitary & $3(4.8)$ & $5(20.8)$ & $5(17.2)$ \\
\hline Acid-fast bacilli smear positivity, $\mathrm{n}(\%)$ & $9(10.7)$ & $7(5.1)$ & $4(4.2)$ \\
\hline NTM species, n (\%) & & & \\
\hline Mycobacterium avium complex & & & \\
\hline avium & $28(46.0)$ & $13(54.2)$ & $9(31.0)$ \\
\hline intracellulare & $12(19.7)$ & $8(33.3)$ & $12(41.4)$ \\
\hline Other avium complex ${ }^{a}$ & $1(1.6)$ & $1(3.4)$ & $1(4.2)$ \\
\hline Mycobacterium abscessus complex & & & \\
\hline Subsp. abscessus & $13(21.3)$ & 0 & $2(6.9)$ \\
\hline Subsp. massiliense & $5(8.2)$ & $2(8.3)$ & $5(17.2)$ \\
\hline Subsp. bolletii & $1(1.6)$ & 0 & 0 \\
\hline Mycobacterium kansasii & $1(1.6)$ & 0 & 0 \\
\hline
\end{tabular}

Data are presented as median (interquartile range) or $\mathrm{n}(\%)$

NTM non-tuberculous mycobacterial; BMI body mass index; COPD chronic obstructive pulmonary disease

${ }^{*} \mathrm{BMI}$ was significantly lower in patients who needed treatment than in patients who were observed without treatment $(P=0.040)$. Fibrocavitary form was more prevalent in patients who received treatment $(P=0.017)$. Among patients who were treated, the BMI was significantly lower in patients with failed treatment than in those who achieved cure $(P=0.024)$

${ }^{\mathrm{a}} M$. marseillense, $M$. colombiense, and $M$. chimaera were isolated

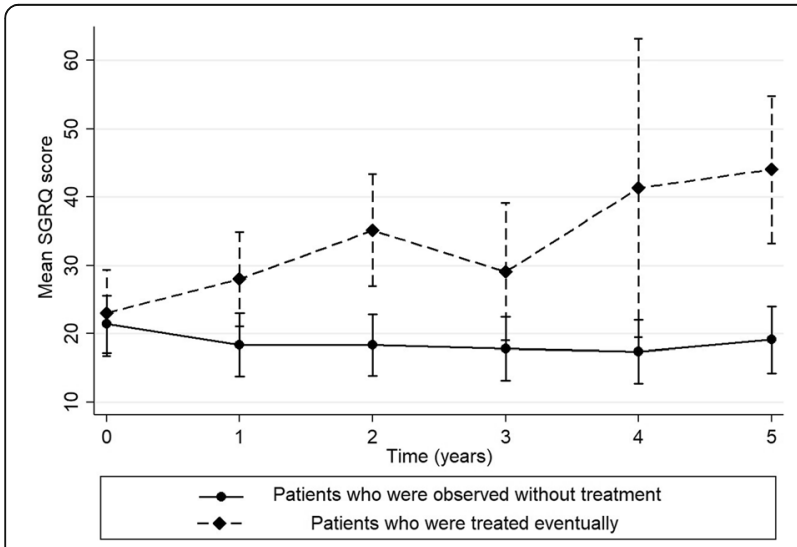

Fig. 2 Mean St. George's Respiratory Questionnaire score with 95\% confidence interval over time according to clinical course during observation period
However, spontaneous conversion did not affect the changes in SGRQ score (coefficient, $-0.76 ; P=0.287$ ).

\section{Changes in SGRQ score with treatment}

During the study period, the patients who achieved a microbiological cure received antibiotic treatment with the median duration of 20 (IQR, 18-24) months. Eight of the study patients (five with M. avium and three with $M$. intracellulare infection) developed recurrence with the same NTM species. Additionally, one patient with $M$. intracellulare developed an $M$. abscessus subsp. abscessus after successful completion of treatment. The patients with treatment failure were treated for the median duration of 24 (IQR, 19-33) months and twelve patients among them were on treatment until the end of the study period. Twelve patients who failed treatment achieved temporary negative conversion of sputum 
Table 2 Association of covariates with treatment initiation

\begin{tabular}{lll}
\hline & Adjusted odds ratio [95\% confidence interval] & $P$-value \\
\hline Age (years) & $1.66[0.95,1.07]$ & 0.789 \\
Female (versus male) & $1.01[0.95,1.08]$ & 0.430 \\
BMI $\left(\mathrm{kg} / \mathrm{m}^{2}\right)$ & $0.94[0.77,1.15]$ & 0.568 \\
Nodular bronchiectatic form (versus fibrocavitary) & $0.79[0.07,8.89]$ & 0.851 \\
Mycobacterium avium complex (versus others) & $1.75[0.50,6.11]$ & 0.378 \\
Baseline SGRQ score & $1.03[0.99-1.07]$ & 0.079 \\
Change of SGRQ score (point/year) & $1.16[1.06,1.27]$ & 0.001 \\
\hline
\end{tabular}

BMI body mass index; SGRQ St. George's Respiratory Questionnaire

culture followed by re-emergence of multiple positive cultures.

The longitudinal changes of SGRQ score according to the treatment outcomes are illustrated in Fig. 3. Among patients who achieved microbiological cure with treatment, the SGRQ score decreased from 33.9 (95\% CI, 26.9-40.9) at the time of treatment initiation to 20.8 (95\% CI, 13.5-28.2) $(P<0.001)$ at 1 year of treatment, to 21.3 (95\% CI, 14.0-28.7) $(\mathrm{P}<0.001)$ at 2 years, and to $17.6(95 \% \mathrm{CI}, 9.1-26.1)(\mathrm{P}<0.001)$ at 3 years. Although patients with treatment failure experienced a decline in the SGRQ score during the first 2 years after initiating treatment (from 36.1 [95\% CI, 28.9-42.5] to 23.1 [95\% CI, 16.6-29.5] at 1 year and to 25.0 [95\% CI, 17.8-32.2] at 2 years), the score at 3 years (30.4) did not decrease. The mean difference at 3 years did not show a significant difference (mean difference, $-5.7[95 \% \mathrm{CI},-14.2$ to 2.7]) (Table 3).

These trends were also observed for the sub-scores of the SGRQ (symptoms, activity, and psycho-social impacts scores). Although the improvements in the symptoms, activity, and impacts scores were maintained until 3 years of treatment in patients who achieved microbiological cure, these scores returned to the baseline levels at 3 years of treatment in patients with treatment failure.

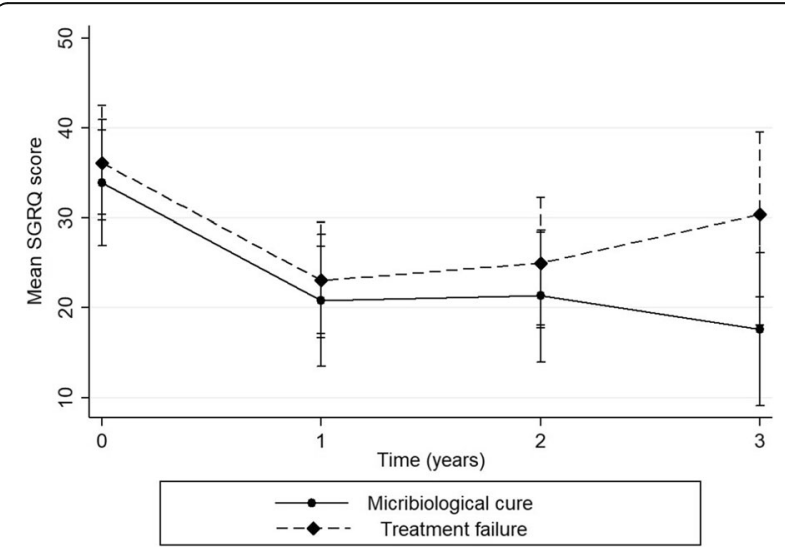

Fig. 3 Mean St. George's Respiratory Questionnaire score with 95\% confidence interval over time with treatment
Changes in SGRQ scores over time were not different in the subgroup of patients who had temporary negative conversion of sputum culture but ultimately failed the treatment (coefficient, $-1.06 ; P=0.873$ ).

\section{Discussion}

We analyzed the longitudinal changes in SGRQ scores according to the clinical course among 114 patients with NTM-PD. While patients who could be observed without treatment showed stationary scores, patients who eventually needed treatment had worsening SGRQ scores during the observational period. Once treatment was initiated, SGRQ scores improved regardless of microbiologic response. However, the improvement did not last among patients who failed in treatment.

The SGRQ was initially developed to measure the health status, including quality of life, in patients with obstructive airway diseases [25]. The SGRQ score could be used as a parameter to assess the treatment responses in patients with bronchiectasis [26] and chronic obstructive pulmonary disease [27]. The SGRQ could also predict the prognosis in patients with chronic obstructive pulmonary disease [28], idiopathic pulmonary fibrosis [29], and chronic pulmonary aspergillosis [30]. Although the HRQL is worse in patients with NTM-PD than in the general population [31], no analysis has been performed to identify the possible association between HRQL and clinical courses of patients with NTM-PD. Our study suggests a role of measuring HRQL using the SGRQ as an additional treatment goal because SGRQ scores can improve even in patients who failed to achieve microbiological cure.

The decision regarding whether to start treatment for NTM-PD has been mainly determined by radiographic or symptomatic changes [6, 7]. However, this decision can be affected by institutions, regions, and nations [32, 33]. The results of this study suggest an association between HRQL and treatment initiation. Although the patients' age, sex, BMI, radiographic type and NTM species did not affect the initiation of treatment, deterioration of HRQL as measured using the SGRQ was observed in patients who needed treatment eventually. 
Table 3 Mean value of St. George's Respiratory Questionnaire score according to treatment outcomes over time

\begin{tabular}{|c|c|c|c|c|c|c|c|c|}
\hline & \multicolumn{2}{|l|}{ Total score } & \multicolumn{2}{|l|}{ Symptoms score } & \multicolumn{2}{|l|}{ Activity score } & \multicolumn{2}{|l|}{ Impacts score } \\
\hline & Mean $(95 \% \mathrm{Cl})$ & $P$-value & Mean (95\% Cl) & $P$-value & Mean (95\% Cl) & $P$-value & Mean $(95 \% \mathrm{Cl})$ & $P$-value \\
\hline \multicolumn{9}{|l|}{ Microbiologic cure } \\
\hline At treatment initiation & $33.9(26.9-40.9)$ & & $47.0(38.9-55.1)$ & & $35.1(25.4-44.8)$ & & $28.5(20.2-36.8)$ & \\
\hline 1 year after treatment initiation & $20.8(13.5-28.2)$ & $<0.001$ & $28.1(19.4-36.9)$ & $<0.001$ & $22.8(12.6-33.1)$ & 0.010 & $15.7(7.0-24.5)$ & 0.002 \\
\hline 2 years after treatment initiation & $21.3(14.0-28.7)$ & $<0.001$ & $31.3(22.8-39.9)$ & 0.001 & $23.0(12.9-33.1)$ & 0.009 & $17.8(9.1-26.4)$ & 0.008 \\
\hline 3 years after treatment initiation & $17.6(9.1-26.1)$ & $<0.001$ & $31.4(20.9-41.9)$ & 0.007 & $21.1(9.3-33.0)$ & 0.015 & $17.1(6.9-27.3)$ & 0.023 \\
\hline \multicolumn{9}{|l|}{ Treatment failure } \\
\hline At treatment initiation & $36.1(29.8-42.5)$ & & $51.7(44.4-59.1)$ & & $37.9(29.1-46.7)$ & & $36.1(28.5-43.6)$ & \\
\hline 1 year after treatment initiation & $23.1(16.6-29.5)$ & $<0.001$ & $36.3(28.8-43.7)$ & $<0.001$ & $26.9(18.0-35.8)$ & 0.007 & $19.0(11.3-26.6)$ & $<0.001$ \\
\hline 2 years after treatment initiation & $25.0(17.8-32.2)$ & 0.001 & $42.6(33.9-51.4)$ & 0.054 & $28.0(18.0-38.1)$ & 0.035 & $24.0(15.4-32.7)$ & 0.003 \\
\hline 3 years after treatment initiation & $30.4(21.2-39.5)$ & 0.184 & $55.0(43.1-66.8)$ & 0.616 & $31.5(18.5-44.5)$ & 0.319 & $26.8(15.6-38.0)$ & 0.096 \\
\hline
\end{tabular}

$\mathrm{Cl}$ confidence interval

This observation suggests the possibility of a change in the SGRQ score as an indicator of the need for treatment initiation among patients with NTM-PD.

The treatment for NTM-PD is lengthy and complicated, and the treatment outcomes are unsatisfactory regardless of species $[8,10]$. In addition, relapse or reinfection after a long duration of treatment has frequently been observed [12, 34]. In this context, a treatment goal other than microbiological cure is required for NTM-PD. In fact, the importance of patient-reported outcomes in future therapeutic trials for NTM-PD has been suggested [35].

Our study showed improvement in HRQL among patients with NTM-PD who were treated. Among patients with NTM-PD cured with treatment, the SGRQ score decreased for 3 years after the initiation of treatment and encompassed all sub-scores of the SGRQ: symptoms, activity, and impact scores. In addition, the improvement in HRQL with treatment was not confined to patients with NTM-PD that had been cured with treatment. Our analysis also showed a decrease in the SGRQ scores for the first 2 years among patients who failed to be cured with treatment. Possible explanation for this is that partial response to treatment, without microbiological cure, could improve patients' symptoms for a while. These observations suggest that improvement in HRQL could be an alternative treatment goal for patients with NTM-PD.

Another issue related to the treatment of NTM-PD is prediction of which patients need and will benefit from antibiotic treatment [35] because antibiotics are associated with the potential for adverse events and the treatment outcome is unsatisfactory [36]. However, reliable indicators of which patients will benefit from therapy are still lacking [35]. This study showed that even in the patients who failed to be cured, HRQL improved for at least 2 years after the initiation of treatment. This observation suggests that active adoption of treatment should be considered among patients with NTM-PD, especially those with worsening HRQL over time.

Our study has several limitations. First, the number of patients with NTM-PD included in this analysis was relatively small. Only one-third of the patients enrolled in our prospective cohort study could be included in this analysis because the others had only one or two yearly SGRQ scores. Second, SGRQ mainly focused on respiratory symptoms and could not reflect the NTM-specific symptoms such as loss of appetite or memory disturbance, which are measured in 'NTM symptom module' [35]. However, most of NTM-PD patients complained of respiratory symptoms [22] and the importance of SGRQ should not be ignored. Third, yearly assessment of SGRQ in this study could dampen the variation of HRQL during the observation period. However, as our study revealed, most of the patients were followed up for a long period and the annual assessment might be more appropriate in real clinical practice.

\section{Conclusions}

In conclusion, worsening HRQL scores were associated with initiation of treatment and, in turn, treatment improved HRQL scores of patients with NTM-PD.
Abbreviations
BMI: Body mass index; HRQL: Health-related quality of life; IQR: Interquartile range; MAC: Mycobacterium avium complex; NTM-PD: Non-tuberculous mycobacterial pulmonary disease; SGRQ: St. George's Respiratory Questionnaire

\section{Acknowledgments}
Not applicable.

\section{Authors' contributions}
J.J.Y. had full access to all of the data in the study and takes responsibility for the integriy of the data and the accuracy of the data analysis. N.K., S.A.K., S.M.C., J.L., and C.H.L. contributed substantially to the study design, data collection, data analysis and interpretation, and the writing and editing of the manuscript. All authors have seen and approved the manuscript. 


\section{Funding}

No funding source.

\section{Availability of data and materials}

The dataset used are available from the corresponding author on reasonable request.

\section{Ethics approval and consent to participate}

This study was conducted in accordance with the amended Declaration of Helsinki and the Institutional Review Board of Seoul National University Hospital approved the protocol (IRB No. 1809-112-974). All patients provided written consents for the study.

\section{Consent for publication}

Not applicable.

\section{Competing interests}

The authors have no conflict of interest to declare.

\section{Author details}

'Division of Pulmonary and Critical Care Medicine, Department of Internal Medicine, Seoul National University College of Medicine, 101 Daehak-Ro, Jongno-Gu, Seoul 110-744, South Korea. ${ }^{2}$ Clinical Trials Center, Seoul National University Hospital, Seoul, South Korea.

\section{Received: 25 October 2019 Accepted: 24 April 2020} Published online: 07 May 2020

\section{References}

1. Lai C-C, Tan C-K, Chou C-H, et al. Increasing incidence of nontuberculous mycobacteria, Taiwan, 2000-2008. Emerg Infect Dis. 2010;16(2):294.

2. Namkoong $H$, Kurashima A, Morimoto $K$, et al. Epidemiology of pulmonary nontuberculous mycobacterial disease, Japan. Emerg Infect Dis. 2016;22(6): 1116-7. https://doi.org/10.3201/eid2206.151086.

3. Henkle E, Hedberg K, Schafer S, Novosad S. Winthrop KLJAotATS. Population-based incidence of pulmonary nontuberculous mycobacterial disease in Oregon 2007 to 2012. Ann Am Thorac Soc. 2015;12(5):642-7.

4. Ringshausen FC, Wagner D, de Roux A, et al. Prevalence of nontuberculous mycobacterial pulmonary disease, Germany, 2009-2014. Emerg Infect Dis. 2016:22(6):1102

5. Lee H, Myung W, Koh W-J, Moon SM, Jhun BW. Epidemiology of nontuberculous mycobacterial infection, South Korea, 2007-2016. Emerg Infect Dis. 2019;25(3):569

6. Griffith DE, Aksamit T, Brown-Elliott BA, et al. An official ATS/IDSA statement: diagnosis, treatment, and prevention of nontuberculous mycobacterial diseases. Am J Respir Crit Care Med. 2007:175(4):367-416.

7. Haworth CS, Banks J, Capstick T, et al. British Thoracic Society guidelines for the management of non-tuberculous mycobacterial pulmonary disease (NTM-PD). Thorax. 2017;72(Suppl 2):ii1-ii64.

8. Kwak N, Park J, Kim E, Lee C-H, Han SK, Yim J-J. Treatment outcomes of Mycobacterium avium complex lung disease: a systematic review and metaanalysis. Clin Infect Dis. 2017;65(7):1077-84.

9. Kwak N, Dalcolmo MP, Daley $\mathrm{CL}$, et al. Mycobacterium abscessus pulmonary disease: individual patient data meta-analysis. Eur Respir J. 2019;54:1801991. https://eri.ersjournals.com/content/54/1/1801991.

10. Diel R, Ringshausen F, Richter E, Welker L, Schmitz J, Nienhaus A. Microbiological and clinical outcomes of treating non-Mycobacterium avium complex nontuberculous mycobacterial pulmonary disease: a systematic review and meta-analysis. Chest. 2017;152(1):120-42.

11. Pasipanodya JG, Ogbonna D, Ferro BE, et al. Systematic review and metaanalyses of the effect of chemotherapy on pulmonary Mycobacterium abscessus outcomes and disease recurrence. Antimicrob Agents Chemother. 2017;61(11):e01206-17.

12. Wallace RJ Jr, Brown-Elliott BA, McNulty S, et al. Macrolide/azalide therapy for nodular/bronchiectatic Mycobacterium avium complex lung disease. Chest. 2014;146(2):276-82.

13. Czaja CA, Levin AR, Cox CW, Vargas D, Daley CL, Cott GR. Improvement in quality of life after therapy for Mycobacterium abscessus group lung infection. A prospective cohort study. Ann Am Thorac Soc. 2016;13(1):40-8.
14. Hama M, Ushiki A, Kosaka M, et al. Health-related quality of life in patients with pulmonary non-tuberculous mycobacteria infection. Int J Tuberc Lung Dis. 2016;20(6):747-52

15. Maekawa K, Ito Y, Oga T, et al. High-resolution computed tomography and health-related quality of life in Mycobacterium avium complex disease. Int J Tuberc Lung Dis. 2013;17(6):829-35.

16. Mehta M, Marras TK. Impaired health-related quality of life in pulmonary nontuberculous mycobacterial disease. Respir Med. 2011;105(11):1718-25.

17. Kim SJ, Yoon SH, Choi SM, et al. Characteristics associated with progression in patients with of nontuberculous mycobacterial lung disease: a prospective cohort study. BMC Pulm Med. 2017;17(1):5

18. Kwak N, Lee $\mathrm{CH}$, Lee $\mathrm{H}$-J, et al. Non-tuberculous mycobacterial lung disease: diagnosis based on computed tomography of the chest. Eur Radiol. 2016; 26(12):4449-56.

19. Clinical \& Laboratory Standards Institute. Interpretive Criteria for Identification of Bacteria and Fungi by DNA Target Sequencing: Approved Guideline, MM18-A. Wayne, PA: CLSI; 2008.

20. Kim B-J, Lee S-H, Lyu M-A, et al. Identification of mycobacterial species by comparative sequence analysis of the RNA polymerase gene (rpoB). J Clin Microbiol. 1999:37(6):1714-20.

21. Salah IB, Adekambi T, Raoult D, Drancourt M. rpoB sequence-based identification of Mycobacterium avium complex species. Microbiol. 2008 154(12):3715-23

22. Park J, Cho J, Lee C-H, Han SK, Yim J-JJCID. Progression and treatment outcomes of lung disease caused by Mycobacterium abscessus and Mycobacterium massiliense. Clin Infect Dis. 2017;64(3):301-8.

23. Kim YS, Byun MK, Jung WY, et al. Validation of the Korean version of the St. George's respiratory questionnaire for patients with chronic respiratory disease. Tuberc Respir Dis. 2006;61(2):121-8.

24. van Ingen J, Aksamit T, Andrejak C, et al. Treatment outcome definitions in nontuberculous mycobacterial pulmonary disease: an NTM-NET consensus statement. Eur Respir J. 2018;51:1800170.

25. Jones PW, Quirk F, Baveystock C. The St George's respiratory questionnaire. Respir Med. 1991;85:25-31.

26. Murray MP, Turnbull K, MacQuarrie S, Hill AT. Assessing response to treatment of exacerbations of bronchiectasis in adults. Eur Respir J. 2009; 33(2):312-8.

27. Brusasco V, Hodder R, Miravitlles M, Lee A, Towse L, Kesten S. Health outcomes in a 6-month placebo controlled trial of once-daily tiotropium compared with twice-daily salmeterol in patients with COPD. Thorax. 2003; 58:399-404.

28. Halpin DM, Peterson S, Larsson TP, Calverley PM. Identifying COPD patients at increased risk of mortality: predictive value of clinical study baseline data. Respir Med. 2008:102(11):1615-24.

29. Furukawa $T$, Taniguchi $H$, Ando M, et al. The St. George's Respiratory Questionnaire as a prognostic factor in IPF. Respir Res. 2017;18(1):18.

30. Lowes D, Al-Shair K, Newton PJ, et al. Predictors of mortality in chronic pulmonary aspergillosis. Eur Respir J. 2017:49(2):1601062.

31. Yeung MW, Khoo E, Brode SK, et al. Health-related quality of life, comorbidities and mortality in pulmonary nontuberculous mycobacterial infections: a systematic review. Respirology. 2016;21(6):1015-25.

32. Adjemian J, Prevots DR, Gallagher J, Heap K, Gupta R, Griffith D. Lack of adherence to evidence-based treatment guidelines for nontuberculous mycobacterial lung disease. Ann Am Thorac Soc. 2014;11(1):9-16.

33. van Ingen J, Wagner D, Gallagher J, et al. Poor adherence to management guidelines in nontuberculous mycobacterial pulmonary diseases. Eur Respir 2017:49(2):1601855

34. Jhun BW, Kim S-Y, Moon SM, et al. Development of macrolide resistance and reinfection in refractory Mycobacterium avium complex lung disease. Am J Respir Crit Care Med. 2018;198(10):1322-30.

35. Henkle E, Aksamit T, Barker A, et al. Patient-centered research priorities for pulmonary nontuberculous mycobacteria (NTM) infection. An NTM research consortium workshop report. Ann Am Thorac Soc. 2016;13(9):S379-S84.

36. Zweijpfenning S, Kops S, Magis-Escurra C, Boeree MJ, van Ingen J, Hoefsloot W. Treatment and outcome of non-tuberculous mycobacterial pulmonary disease in a predominantly fibro-cavitary disease cohort. Respir Med. 2017; 131:220-4.

\section{Publisher's Note}

Springer Nature remains neutral with regard to jurisdictional claims in published maps and institutional affiliations. 